Barber, M. (1953). J. gen. Microbiol. 8, 111-115.

\title{
Penicillin-resistant and Penicillin-dependent Staphylococcal Variants
}

\author{
BY MARY BARBER \\ Department of Bacteriology, St Thomas's Hospital Medical School, London
}

SUMMARY: Four strains of Staphylococcus aureus (coagulase-positive) were each subjected to three or more series of transfers on penicillin ditch plates. The plates were examined for colonial variants and four quite distinct types of penicillinresistant variant, one of which was penicillin-dependent, were isolated. These types often occurred in association, and sometimes as many as three were isolated from a single culture plate. The main characteristics of the different types are described.

Studies on the incidence of penicillin-resistant bacterial variants began soon after the isolation of the antibiotic and have continued in increasing numbers (see Abraham et al. 1941; Todd, Turner \& Drew, 1945; Demerec, 1945, 1948; Bellamy \& Klimek, 1948; Gale \& Rodwell, 1948; Hughes, 1952). In most studies of this description bacteria have been subcultured in fluid medium containing increasing concentrations of penicillin. In the present investigation solid medium was used to determine whether different types of variant could be isolated from the same culture.

\section{METHODS}

Strains of staphylococci. Four strains of Staph. aureus were studied; two strains had been isolated from infectious processes and were phage-type 3B/3C (strain D14) and 6/47 (strain STH10) respectively; two strains had been isolated from the noses of nurses in a maternity department and were phage-type 52A (strain M109) and 6/47+ (strain M12).

Penicillin. Crystalline penicillin G (Glaxo) was used throughout.

Passage in the presence of penicillin. The four strains were serially transferred on penicillin ditch plates. The medium used consisted of $1 \%$ 'LabLemco', $1 \%$ peptone, $0.5 \% \mathrm{NaCl}$ and $2 \%$ Bacto shred agar. Ditches were cut at one side of the plate and filled with the same medium containing at first 10 and later $100 \mathrm{u}$. penicillin $/ \mathrm{ml}$. The ditch plates were stored at room temperature for $\mathbf{2 4} \mathrm{hr}$. before use, and subcultures made from the edge of growth nearest to the ditch at 4- to 7-day intervals. Each strain was subjected to three or more such series of transfers.

Sensitivity to penicillin of the parent and variant cultures was estimated by the serial dilution method in 'Lab-Lemco' broth. All the parent cultures were sensitive to from 0.05 to $0.01 \mathrm{u} . / \mathrm{ml}$.

Penicillinase production was measured by the cylinder plate method. Young broth cultures of the variants to be tested were mixed with equal quantities of penicillin dissolved in distilled water to give final concentrations of 1-5u. penicillin $/ \mathrm{ml}$. The mixtures were incubated at $37^{\circ}$ for $2-24 \mathrm{hr}$. before transferring to the cylinders. 
Inactivation of penicillin. (1) With penicillinase: preparation of penicillinase (Burroughs Wellcome), $1 \mathrm{ml}$. of which inactivated 100,000u. of penicillin, was used; $0.1 \mathrm{ml}$. was added to $1000 \mathrm{u}$. of penicillin in $10 \mathrm{ml}$. distilled water and the mixture left to stand at room temperature for some hours before use.

(2) Acid treatment. Ten ml. 0.01 N-HCl ( $\mathrm{pH} \mathrm{2.0)}$ was added to an ampoule containing $100,000 \mathrm{u}$. penicillin and the mixture kept at $37^{\circ}$ for $3-6 \mathrm{hr}$. Tests were carried out with the equivalent of $1 \mathrm{u} . / \mathrm{ml}$. penicillin in nutrient agar; at this dilution the acid did not affect the $\mathrm{pH}$ of the medium.

\section{RESULTS}

Four quite distinct types of penicillin-resistant variant were encountered; they often occurred in association and sometimes as many as three were isolated from a single culture plate. Types 1 and 2 were the most frequently isolated, but, since they grew more luxuriantly than types 3 and 4 , this does not necessarily mean that they occurred with greater frequency. An attempt was not made to measure the actual frequency of the variants.

Type 1 variant. This type of variant was isolated from all four strains of Staph. aureus and tended to overgrow other types. Its main characteristics are illustrated in Pl. 1, fig. 1. It will be seen that the variant gives typical staphylococcal growth over that part of the plate permitting growth of the parent culture; over the rest of the plate, including the penicillin ditch, growth also occurs, but is less luxuriant and semi-transparent. Gram films taken from opposite halves of the plate also showed a difference. The organisms growing some distance away from the penicillin appeared to be typical staphylococci with a fairly uniform appearance, whereas the organisms growing in the neighbourhood of the penicillin ditch showed gross variations in size and staining, with many large swollen and deeply Gram-positive cells. When subcultures were made on to fresh ditch plates, the same picture resulted, irrespective of which part of the plate the subculture came from.

This type of variant appeared to develop resistance to penicillin gradually and continuously with each transfer, until a culture able to grow in $100 \mathrm{u} . / \mathrm{ml}$. or more was obtained. With strain M12, after five transfers a variant of this type appeared which grew to the edge of a ditch containing $10 \mathrm{u} . / \mathrm{ml}$, , after ten transfers the variant grew right across such a ditch, and after twenty transfers the variant grew right across a ditch containing $100 \mathrm{u} . / \mathrm{ml}$. Intermediate degrees of resistance were seen at intervening periods. When such a penicillin-resistant variant was serially transferred in broth without penicillin there was a similarly gradual decrease in resistance, until the culture became as sensitive to penicillin as the parent.

Type 2 variant. Variants of this type occurred very frequently in association with those of type 1 . They differed from the latter in that they grew uniformly right across penicillin ditch plates. The degree of growth was variable, but it was usually less luxuriant than that of the parent strains. Gram films showed organisms which were fairly regular in size, shape and staining, but some swollen deeply Gram-positive cells were usually present. Two variants of type 2 are seen in Pl. 1, fig. 2, and variants of types 1 and 2 isolated from 
a single culture plate are shown in $\mathrm{Pl}$. 1, fig. 3. Type 2 variants were isolated from strains D14, STH10 and M12. Their origin, degree of resistance and stability were similar to those of type 1 .

Type 3 variant. These are small colony variants resembling $g$ forms. They showed a slight or moderate increase in resistance to penicillin, which was not increased by further passage in the presence of the antibiotic or decreased by transfer in its absence. This type of variant appeared in a single stage, suggesting a one-step mutation. The variant of this type seen in Pl. 1, fig. 4, was able to grow in a concentration of penicillin sixteen times that which permitted growth of the parent culture. In Gram films of this variant most of the organisms were typical staphylococci, but a few elongated cocci were seen.

Type 4 variant. These variants were found to be penicillin-dependent; maximum growth occurred in the presence of 0.05-1.0u. penicillin $/ \mathrm{ml}$., i.e. about the minimum concentration of penicillin which inhibited the parent cultures. Even in the presence of the optimum concentration of penicillin growth was feeble and on solid medium the colonies were pin-point and semitransparent. A typical example is shown by itself in Pl. 2, fig. 5, and mixed with the parent culture in Pl. 2, fig. 6. In the latter picture it will be seen that there is a slight gap, containing ghost-like colonies only, between the growths of the parent and variant strains. Gram films of this variant showed a moderate amount of variation in morphology and staining of the cells and some large deeply Gram-positive cocci.

This type of variant was isolated only from strains D14 and M109. In some experiments with these strains, after ten to twenty-five transfers on penicillin ditch plates, all or nearly all the surviving staphylococci appeared to be penicillin-dependent (see Pl. 2, fig. 7). In other experiments variants of this type were selected from plates showing a variety of penicillin-resistant types. Pl. 2, fig. 8, shows variants of types 1 and 4 selected from a single culture plate. The penicillin-dependent variants were extremely unstable, and even in the presence of penicillin tended to yield colonies resembling the parent culture or type 1 variants. Intermediate colonies were not isolated.

Experiments were carried out to determine whether these variants would grow when penicillin was replaced by inactivated preparations. For this, plates were prepared with nutrient agar (1) without addition, $(2)+1 \mathrm{u}$. penicillin $/ \mathrm{ml}$., (3) $+1 \mathrm{u}$. penicillin $/ \mathrm{ml}$. inactivated with penicillinase, and $(4)+1 \mathrm{u}$. penicillin $/ \mathrm{ml}$. inactivated by acid. The results of such an experiment are shown in Pl. 3, fig. 9. The plates were heavily seeded on one half with the parent strains, and on the other half with the penicillin-dependent variant. It will be seen that, apart from the site of heavy inoculum, the variant only grew on the plate containing active penicillin, whereas the parent culture did not grow on this plate, but grew on the other three.

\section{Penicillinase production}

Cultures from all four strains were tested for their capacity to produce penicillinase after eight, ten and seventy-five transfers on penicillin ditch plates. Significant destruction of penicillin was not detected. The method 
employed, however, was not sufficiently sensitive to detect the destruction of very small amounts, such as may have been utilized by the penicillindependent variants.

\section{DISCUSSION}

It is clear from these experiments that penicillin-resistant staphylococcal variants may be of many different kinds. Four have been described here, and as many as three were derived from a single strain. Thus mathematical studies, seeking merely to enumerate the cells in a given population able to grow in a given concentration of penicillin, may well be misleading, and can only be used with reserve as a guide to the genetical behaviour of bacteria.

Dogmatic statements cannot be made about the mode of origin of the four types of variant here described. In types 3 and 4, the suddenness of appearance and lack of intermediate types suggests that these variants are mutants. The characteristics of type 1 variants, however, suggest a different mode of origin. With these variants increase in resistance to penicillin occurs gradually, resembling the type of penicillin-resistant staphylococcal variants described by Hughes (1952). Further, these variants lose their increased resistance to penicillin in a gradual fashion when cultivated in the absence of the antibiotic. Both the gain and loss in resistance appear to be a slow continuous change. These features might be compatible with step-wise mutation described by Demerec (1948) but in that case, as Hughes (1952) points out, the number of steps must be very great. Change of an adaptive nature would, therefore, seem a more probable explanation for the emergence of this type of variant.

Nearly all the penicillin-resistant strains of Staph. aureus isolated from infective processes are resistant because they produce penicillinase (see Barber, 1949). This type of resistant variant was not encountered in these experiments. Most other investigators have also found that penicillin-resistance acquired by staphylococci in vitro is not associated with penicillinase production. Bellamy \& Klimek (1948) describe a strain trained in vitro to grow in $4 \mathrm{mg}$. penicillin $/ \mathrm{ml}$., which produced small amounts of penicillinase, but only in the presence of penicillin. This strain, however, unlike the penicillin-destroying strains so frequently isolated in vivo, had lost many of the properties of a typical staphylococcus. It is unlikely that any of the penicillin-resistant variants here described would be of importance in clinical practice since they would readily be overgrown by typical penicillin-sensitive strains.

My thanks are due to Mr A. L. Wooding for the photographs.

\section{REFERENCES}

Abraham, E. P., Gardner, A. D., Chain, E., Heatley, N. G., Fletcher, C. M., Jennings, M. A. \& Florey, H. W. (1941). Further observations on penicillin. Lancet, ii, 177.

BARBER, M. (1949). The incidence of penicillin-sensitive variant colonies in penicillinase-producing strains of Staphylococcus pyogenes. J. gen. Microbiol. 3, 274.

Bellamy, W. D. \& Klimek, J. W. (1948). Some properties of penicillin-resistant staphylococci. J. Bact. 55, 153. 
Journal of General Microbiology, Vol. 8, No. 1
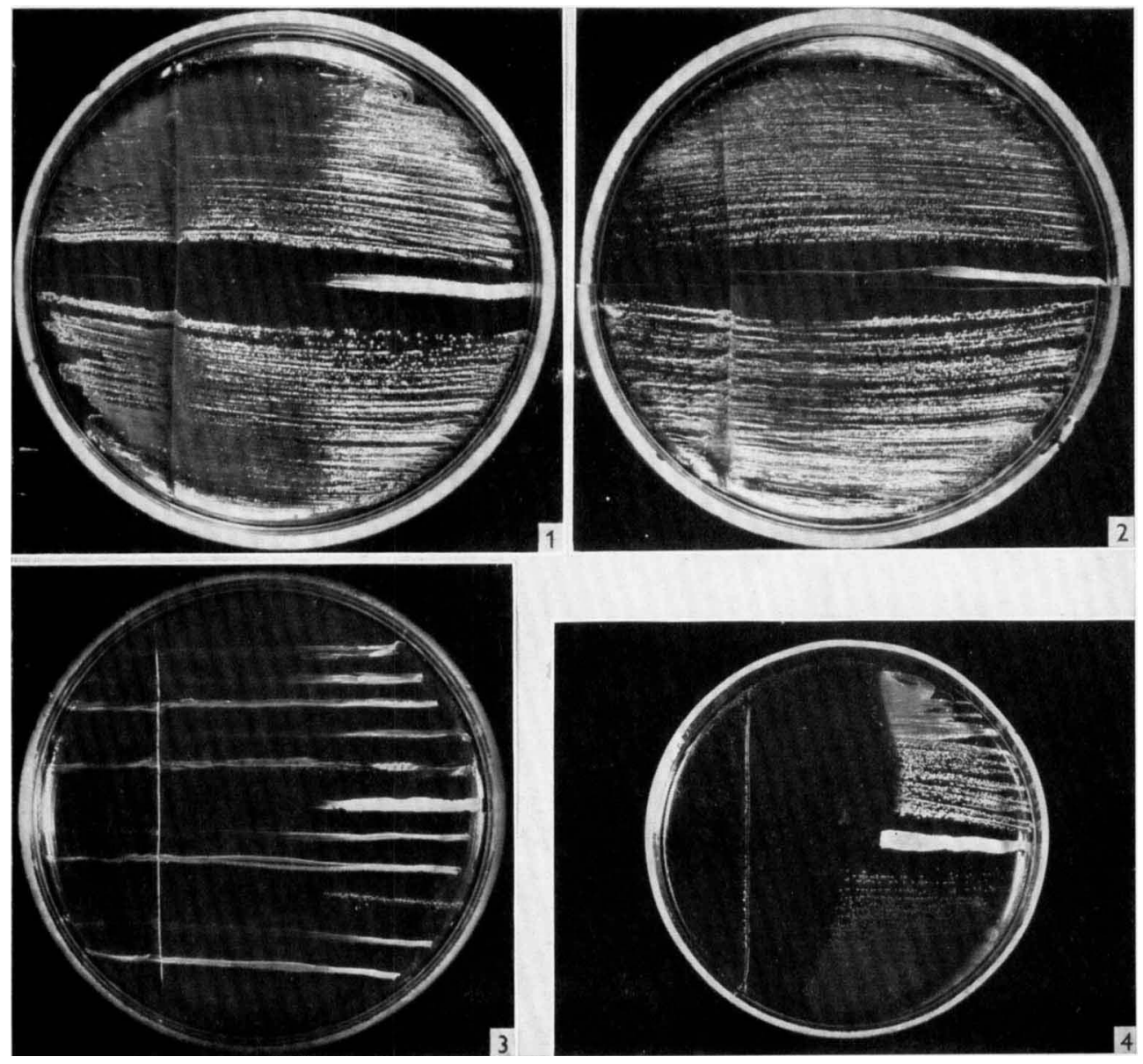

M. Barber-Penicillin-dependent stapilylococci. Piate 1 
Journal of General Microbiology, Vol. 8, No. 1
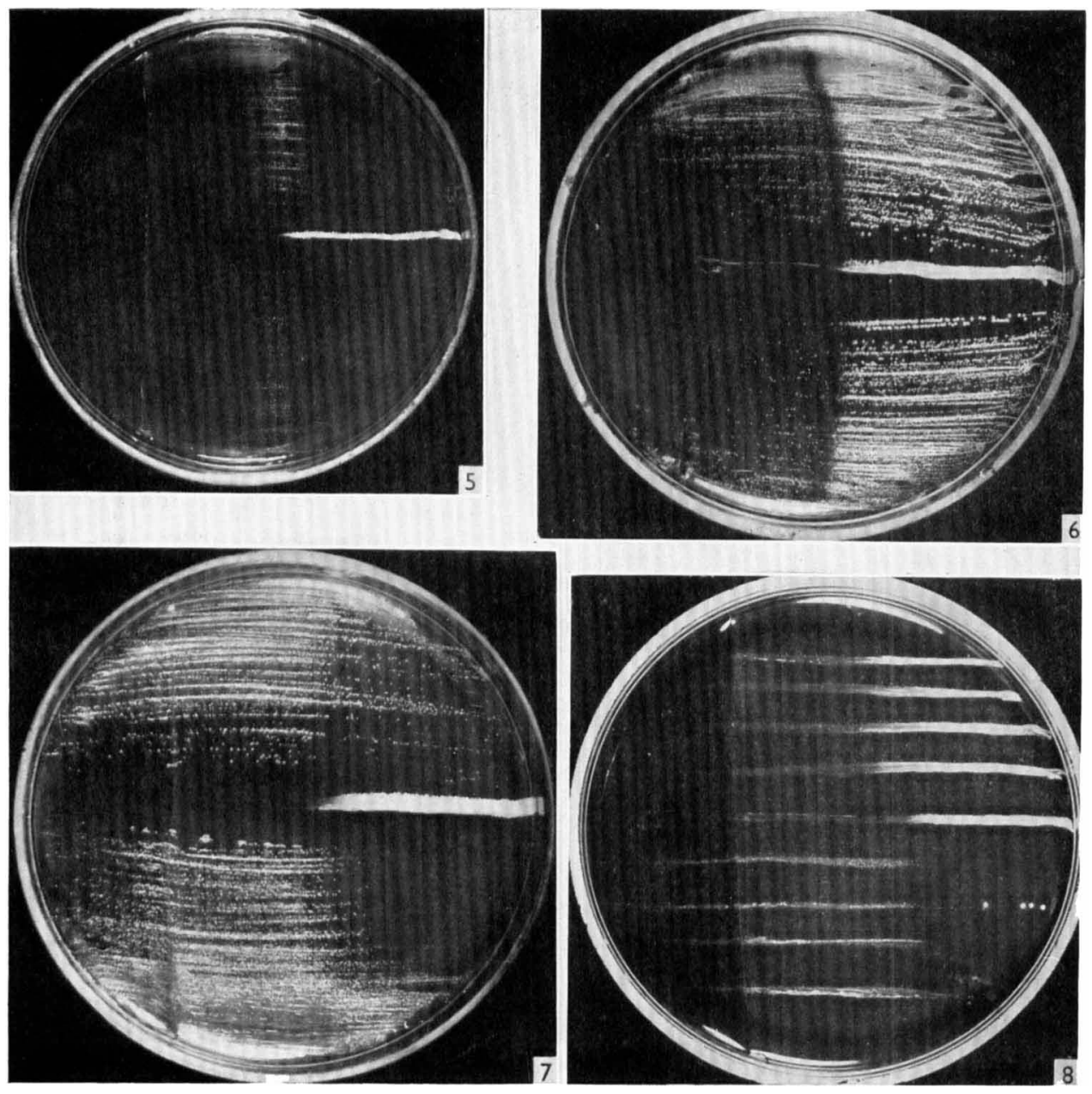

M. Barber-Penicillin-dependent staphylococci. Plate 2 
Journal of General Microbiology, Vol. 8, No. 1
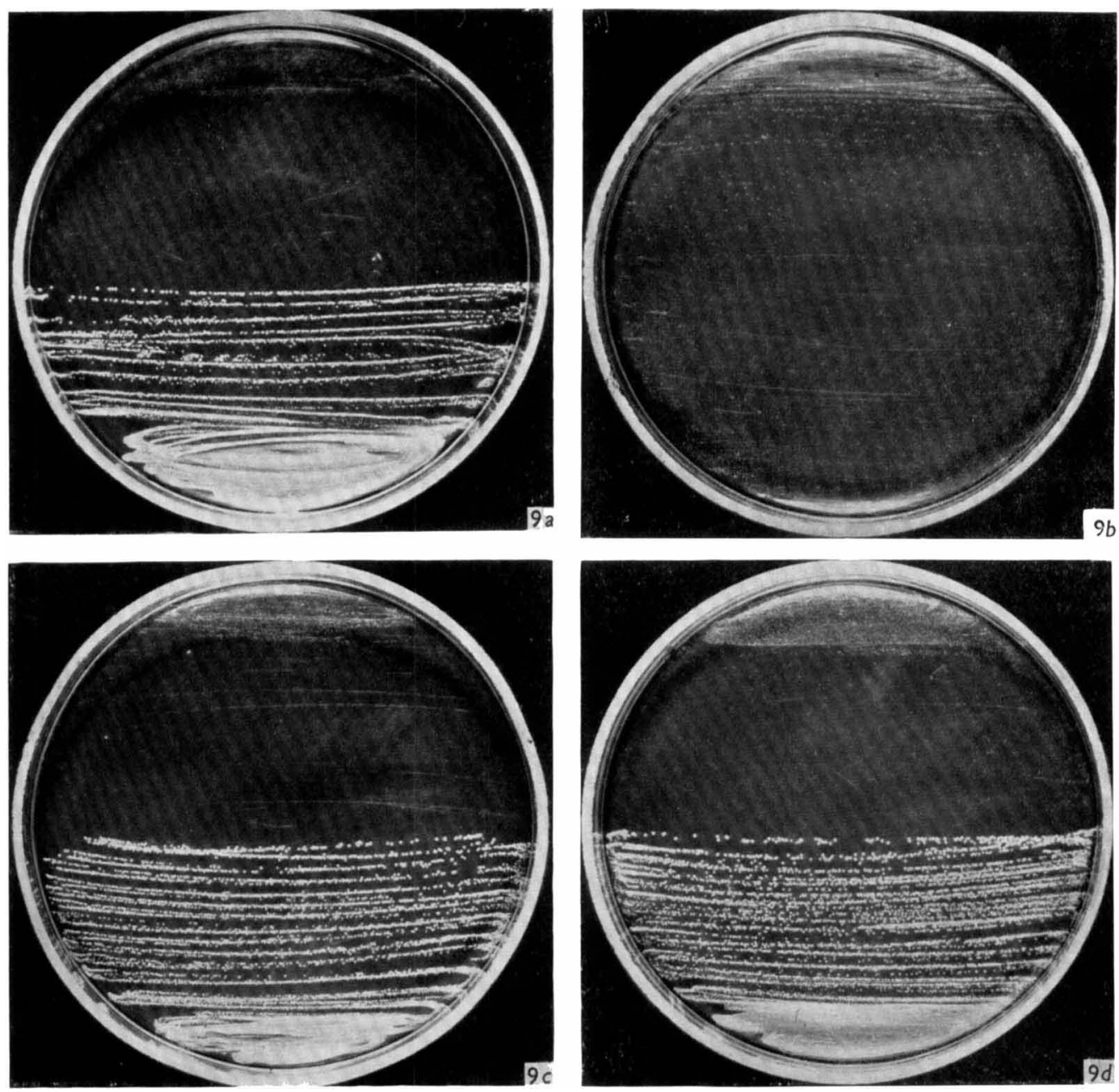

M. Barber--Penicillin-dependent stapiylococct. Peate 3 
Demerec, M. (1945). Production of staphylococcus strains resistant to various forms of penicillin. Proc. nat. Acad. Sci., Wash. 31, 215.

Demerec, M. (1948). Origin of bacterial resistance to antibiotics. J. Bact. 56, 63.

Gale, E. F. \& Rodwell, A. W. (1948). Amino-acid metabolism of penicillinresistant staphylococci. J. Bact. 55, 161.

Hughes, W. H. (1952). Variation in penicillin resistance in single-cell cultures of Staphylococcus aureus. J. gen. Microbiol. 6, 175.

Todd, E. W., Turner, G. S. \& Drew, L. G. W. (1945). The temporary character of 'fastness' of staphylococci to penicillin. Brit. med. J. i, 111.

\section{EXPLANATION OF PLATES 1-3}

Figs. 1-8. Penicillin ditch plates; ditch contains $10 \mathrm{u}$. penicillin $/ \mathrm{ml}$.

\section{Plate 1}

Fig. 1. Type 1 variant derived from strain M12 after eighty-nine transfers. Central streak represents parent culture.

Fig. 2. Type 2 variants derived from strains STH10 $(a)$ and $M 12(b)$ after twenty and twenty-five transfers respectively. Central streak represents parent culture of strain STH 10.

Fig. 3. Streaks represent single colonies derived from strain D 14 after forty-six transfers. Five streaks represent type 1 variants, four type 2 and one type 3 . Central streak represents parent culture.

Fig. 4. Lower half shows a type 3 variant derived from strain STH10 after twenty-five transfers. Culture on upper half and central streak represent parent culture.

\section{Plate 2}

Fig. 5. Type 4 variant derived from strain D14 after eleven transfers. Central streak represents parent culture.

Fig. 6. Same variant as shown in fig. 5 mixed with parent culture. Central streak represents parent culture.

Fig. 7. Predominance of type 4 variants occurring after strain $M 109$ had been transferred twenty-five times. Central streak represents parent culture.

Fig. 8. Streaks represent different colonies derived from strain D14 after eleven transfers. Four streaks represent type 1 variants and four type 4. Central streak represents parent culture.

\section{Plate 3}

Fig. 9. Type 4 variant derived from strain D14 after thirty-four transfers and parent culture plated out on: $(a)$ nutrient agar alone; $(b)$ nutrient agar + penicillin $(1 \mathrm{u} . / \mathrm{ml}$.$) ;$ (c) nutrient agar + penicillin $(1 \mathrm{u} . / \mathrm{ml}$.) inactivated by penicillinase; $(d)$ nutrient agar + penicillin $(1 \mathrm{u} . / \mathrm{ml}$.) treated with $\mathrm{HCl}$ at $\mathrm{pH} 2 \cdot 0$.

(Received 18 July 1952) 